\title{
El fundamento y el uso de las clasificaciones científicas: dinámicas desfasadas
}

María L. Christiansen Renaud Universidad de Guanajuato

\section{Resumen}

Este artículo plantea de forma sucinta problemas epistemológicos ligados a las prácticas clasificatorias de la psiquiatría y la psicología, al revisar de modo crítico algunas de las premisas del movimiento antipsiquiátrico en su ofensiva contra el modelo naturalista de la psicología médica. En el intento abordar el debate desatado entre realistas y constructivistas en torno a la noción de enfermedad mental y sus derivaciones taxonómicas se expone la tesis propuesta por el filósofo e historiador Ian Hacking bajo la expresión de un bucle de retroalimentación entre los médicos (clasificadores), los pacientes (clasificados) y los sistemas de conocimiento (marcos categoriales). Por último se proyectan las ideas analizadas hacia un enfoque dominante en el terreno de la psicología actual: la psicometría, con el propósito de explorar, en este caso particular, el rendimiento explicativo de las posturas epistemológicas presentadas.

Palabras clave: psiquiatría, antipsiquiatría, clasificación, enfermedad mental, bucle de retroalimentación, psicometría.

\section{Abstract}

This paper sets out epistemological problems raised by psychological and psychiatric classificatory practices, and it examines critically some premises supported by antipsychiatric movement against the psychiatric naturalism. 
In order to tackle the heated debate about the Reality / Construction of mental disorders - and its taxonomical implications - the famous Ian Hacking's thesis about the looping effect derived from classificatory practices is proposed and analyzed. Finally, the exposed ideas are applied to Psychometry, a prevalent tendency at the present psychology. The aim is to evaluate the explanatory fertility of the epistemological positions established in this paper.

Keywords: psychiatry, antipsychiatry, classification, mental illness, looping effect, psychometry.

\section{Introducción}

A lo largo del siglo XX hemos asistido a una situación particular: Aciencias como la psiquiatría y la psicología se han instalado en nuestra cultura no sólo en términos de autoridades epistémicas capaces de explicar las conductas humanas sino como ámbitos generadores de un "servicio profesional" por medio del cual un científico puede hacer, mejor que nadie, lo que antes era de la competencia de la religión o del consejero moral: curar el alma, corregir los defectos del carácter, modificar la conducta, cambiar el destino, enseñar a vivir. En nuestro lenguaje común nos referimos a la enfermedad mental como si se tratara de una cosa natural, de una clase de entidad de existencia autónoma, dada. Lo mismo sucede con el concepto más amplio de conducta anormal, que en el imaginario popular es usado con frecuencia para clasificar una multiplicidad de formas de comportamiento que parecieran caer por naturaleza dentro o fuera del territorio de lo normal. El concepto de normalidad es, en tal sentido, un término meta-clasificatorio, porque clasifica clasificaciones. Dicho de otro modo, sea cual fuere la forma en la cual se clasifica una conducta humana, podemos estar seguros de antemano que la clasificación por la que optemos será, a su vez, subsumida bajo las categorías más 
amplias de condición normal o anormal (por ejemplo, el desorden de hiperactividad y déficit de atención clasifica un modo de conducta específica, pero a su vez es él mismo clasificado bajo el conjunto de conductas anormales).

Sin embargo, y esto es lo que resulta intrigante, esa simplicidad con la que hablamos de tales entidades en el discurso cotidiano contrasta con lo que sucede en el discurso epistemológico. Pues así como el siglo XX ha atestiguado la naturalización de los conceptos de enfermedad mental y conducta anormal en el lenguaje común, también ha atestiguado la batalla campal que, sobre dichas nociones, ha tenido lugar en los debates académicos de los psiquiatras, los psicólogos, los psicoanalistas y los sociólogos. Recorramos un poco el siglo XX y veamos cómo ha estado el panorama.

\section{Un mosaico de posturas en controversia: psiquiatría / antipsiquiatría}

Comenzaré por las dos primeras décadas, tomando como punto de partida el caso del concepto de histeria y, en consecuencia, me ubicaré en Francia para referirme a Joseph Babinski, el más brillante de los alumnos de Charcot cuando éste era el más destacado de los neurólogos parisinos (al punto de haber sido bautizado como "el padre de la histeria”). A pesar de haber sido su alumno, Babinski presentó ante la Sociedad de Neurología en 1908 una tesis según la cual la histeria no se debía a ningún daño neurológico, con lo que refutaba a Charcot. La hipótesis de Babinski establecía que los fenómenos histéricos eran el producto de la sugestión de ideas inducida por el médico y absorbida por canales culturales. Este ataque de Babinski a la concepción charcotiana de la histeria contribuyó a la desaparición de ésta y los síntomas que la tipificaban se redistribuyeron en un nuevo conjunto de enfermedades. Lo que quiero resaltar al mencionar este caso es que la extinción de la histeria como forma de diagnóstico fue mucho 
más ágil en la comunidad científica que en el lenguaje común, donde aún hoy hay remanentes conceptuales. La taxonomía psiquiátrica ya no fue la misma después de la desaparición de la histeria, pero, en el lenguaje popular el concepto de histeria y ciertas imágenes e ideas asociadas a tal noción no han desaparecido de manera completa.

Ahora bien, una influencia que también fue importante para la desaparición de la histeria como diagnóstico médico fue el hecho de que, en la época, se estaban imponiendo nuevos conceptos y formas de clasificación, como la de demencia precoz, procedente de Alemania, que en poco tiempo fue reemplazada por la de esquizofrenia. Y aquí nos enfrentamos a un nuevo ejemplo de cómo las controversias paradigmáticas en las comunidades científicas no impide la infiltración, propagación y conservación de los conceptos en el lenguaje común. El debate sobre el caso de la esquizofrenia estalló en la década de 1960, cuando llegó a consolidarse el denominado movimiento antipsiquiátrico, encabezado por David Cooper en Inglaterra, el cual tenía el firme propósito de mostrar que no existía ningún territorio epistemológico en el cual la psiquiatría cumpliera una función cognoscitiva legítima. Para los antipsiquiatras estaba claro que las llamadas enfermedades mentales no existían. O bien eran enfermedades neurológicas, o condiciones genéticas o desordenes bioquímicos (y por lo tanto del dominio del neurólogo, del geneticista o del bioquímico); o bien eran formas de conducta relacionadas con la historia personal de un individuo ( $y$, en consecuencia, del dominio del psicólogo). Así, el psiquiatra no tenía ningún espacio en el mapa de las profesiones. En el enfrentamiento entre quienes seguían defendiendo la legitimidad de la psiquiatría y quienes la condenaban, el concepto de esquizofrenia era el blanco de discusión. De hecho aparecía en el título de varias de las obras de los antipsiquiatras (por ejemplo, en la de G. Deleuze y F. Guattari, Anti-Edipo. Capitalismo y Esquizofrenia, de 1972). Desde la óptica de los antipsiquiatras el caso de la esqui- 
zofrenia formaba parte de la tendencia generalizada a medicalizar las desviaciones sociales en términos de locura, lo cual instauraba una nueva forma de opresión (comparable a la de la Inquisición, pero reemplazando el mal por la enfermedad, los argumentos teológicos por los psiquiátricos y la conversión forzada de herejes por la internación obligatoria). Aunque este movimiento antipsiquiátrico no perduró en su forma original, el escepticismo acerca de la esquizofrenia como una forma de diagnóstico sigue vigente, como lo ilustra, por ejemplo, el título de un libro de Mary Boyle que retoma este interrogante: Esquizofrenia: ¿un fraude científico? (1990). Aquí la situación vuelve a ser similar. Luego del huracán antipsiquiátrico la esquizofrenia sigue siendo una forma de diagnóstico usada en este caso por la comunidad científica y también presente en el lenguaje común.

Otros conceptos han corrido una suerte parecida. Como nos muestra Ian Hacking en Rewriting the Soul, en la década de 1980 se desató la tormenta académica en torno al concepto de desorden de personalidad múltiple, enfrentando a quienes pretendían incluir esta forma de diagnóstico en el manual de clasificación de uso estándar en Estados Unidos, el Diagnostic and Statiscal Manual of Mental Disorder, y quienes se oponían a aceptar su inclusión (la cual se logró en 1987). Otros conceptos del ámbito psiquiátrico y psicoterapéutico han sido igualmente debatibles, como los de autismo, desorden de fatiga crónica, anorexia y bulimia, entre otros. En el debate no hallamos una negación de que representen trastornos de la conducta, lo que se ha discutido es que sean enfermedades. Es decir, al dudar de su realidad, lo que se cuestiona es su fundamento ontológico, su realidadcomo-enfermedad. Uno de los argumentos principales para negarles realidad ha sido el de la imposibilidad de mostrar que estas formas de diagnóstico sean generalizables, transculturales. Los desórdenes alimentarios, las obsesiones con los parámetros estéticos, la adicción al trabajo, las afecciones relacionadas con el miedo y la soledad, etc., 
parecieran ser, según los escépticos, manifestaciones de una forma de vida típica de la cultura occidental, mientras que, si fuesen enfermedades reales, no presentarían estas características locales. No obstante estas tendencias de incredulidad, los conceptos de personalidad múltiple, anorexia y bulimia, autismo, paranoia, fobia, fatiga crónica, y otros desórdenes que se hallan en situación análoga, son formas en las cuales la gente común se refiere a su propia conducta y a la conducta de los demás; tales nociones pueblan, como entidades lingüísticas, nuestro lenguaje cotidiano y se instalan cómodamente en nuestros imaginarios sociales.

Así, a lo largo del siglo XX se ha ido formando un paisaje muy heterogéneo en cuanto a los modelos explicativos de las conductas anormales, pero esa heterogeneidad propia de la academia no es percibida como tal fuera de la academia, donde la perspectiva psiquiátrica, que se presenta como una rama de la medicina, sigue ejerciendo el monopolio de la verdad de los fenómenos del comportamiento y de la salud mental. Ahora, como ya hemos advertido, esa hegemonía ha tenido, y tiene, sus aliados y también sus rivales. Mientras que la psiquiatría tiende a ver las conductas anormales como consecuencias de enfermedades mentales, el modelo psicodinámico le niega tal status y la concibe como un desajuste mental causado por conflictos intrapsíquicos originados en la infancia. En oposición, el modelo conductista ha definido la conducta anormal como una respuesta inadaptada obtenida por un condicionamiento no-exitoso, mientras que el modelo intencional la define como una forma de acción racional y deliberada con la cual el actor persigue un fin determinado. Algunos sociólogos han rechazado todas estas formas de explicación argumentando que la conducta anormal tiene un origen externo al individuo, ya que es causada por fuerzas sociales en las que se halla inmerso (la familia, las instituciones). 
El espectro de posiciones que orbitan en torno a la salud mental es tan complejo que podríamos coincidir con la apreciación de Hacking cuando, parafraseando al Wittgenstein de las Investigaciones Filosóficas, sostiene que:

En la psicología tenemos los métodos clínicos de la medicina, la psiquiatría, la psicología, las innumerables variantes del psicoanálisis, los sistemas de autoayuda, la terapia de grupos, los consejeros, incluyendo al sacerdote y al gurú; tenemos igualmente los métodos estadísticos de la epidemiología y la genética poblacional, los métodos experimentales de la bioquímica y la neurología, la patología y la biología molecular, la modelación teórica de la ciencia cognitiva y CONFUSIÓN CONCEPTUAL (Hacking, 1995).

Realidad / Construcción de las enfermedades mentales: bucles de retroalimentación

¿Son reales las enfermedades mentales? ¿Qué significa enfermedad mental? ¿Qué significa realidad para los científicos? ¿Qué significa realidad para el sentido común? Cada uno de estas interrogantes conduce a laberintos semánticos y epistemológicos de profundas consecuencias para la práctica y la intervención científica. Como hemos podido apreciar con los ejemplos anteriores, los conceptos que, en determinado momento, han sido parte del discurso científico (histeria, esquizofrenia, fugas demenciales, personalidades múltiples (o, como se lo llama hoy: disociación), interactúan con el lenguaje común, no científico, en múltiples modos. Una vez que el uso de un concepto se estabiliza dentro del universo de discurso de una comunidad científica, dejando de ser un paradigma para convertirse en un prototipo (distinción de Kuhn), ese concepto que tiene en la jerga científica una acepción definida, claramente demarcada, se divulga, se extiende y deviene un objeto de interés cultural, pasa a ser con pro- 
babilidad un ingrediente de la literatura, del cine, de los talk shows, de la retórica forense, de la aparición de sociedades de ayuda a quien padece de esa nueva enfermedad, y poco a poco la gente comienza a usarlo para describir acciones de modos en los que antes no era posible describirlas.

En tal sentido, un nuevo concepto científico, una nueva forma de clasificación de conductas humanas nos coloca, tomando las palabras de Kuhn, "en un mundo nuevo". La emergencia de un nuevo concepto hace posible reordenar de un modo por completo distinto un sector de la experiencia humana. Y cuando esto ocurre es posible ver las conductas individuales desde una óptica innovadora. Retomo a Hacking para ilustrar estas afirmaciones con un ejemplo: el del concepto de abuso infantil. Como una forma de experiencia, tenemos que decir que el núcleo de vivencias que hoy englobamos bajo el nombre de abuso infantil ha existido a lo largo de la historia, pero como una forma particular de clasificar ciertas conductas el concepto de abuso infantil es relativamente nuevo. Incluso en las primeras décadas del siglo XX tenía sentido hablar de maltrato infantil o de incesto, pero no de abuso infantil. Cada uno de esos conceptos ha llegado a implicar diferentes cosas. Por ejemplo, cuando se hablaba de maltrato por lo general se hacía referencia a bebés maltratados, mientras que la noción de abuso es más abarcadora en cuanto a la edad de la víctima, y la noción de incesto aludía a una situación sexual, mientras que el abuso es un concepto más híbrido que no se puede reducir al abuso sexual, ya que incluye abuso verbal, emocional, etc. Pero, además, este concepto de abuso infantil no ha surgido aisladamente, sino de la mano de una red de transformaciones conceptuales que le han dado un lugar muy importante en la psicología, en particular el psicoanálisis. Por ejemplo, la noción de abuso infantil está íntimamente relacionada con la idea de trauma (entendido éste último en el sentido de una lesión psicológica) y ambas se han entretejido en teorías causales, 
como por ejemplo la que asume que las repetidas experiencias de abuso durante la infancia son causa de trauma y que éste afecta de manera fundamental a la memoria de la víctima, al producir cierta clase de amnesia que conduce a enterrar un pasado doloroso. El punto al que quiero llegar —en el seguimiento del análisis anticipado por Hacking - es el siguiente: antes de la existencia de la categoría de abuso infantil ¿cómo eran clasificadas esas experiencias? Hoy tiene sentido decir que una persona $\mathrm{X}$ sufrió abuso durante su infancia. Esto sucede con mucha frecuencia en la psicoterapia, cuando el paciente llega a descubrir que fue abusado por un familiar o un allegado o cualquier otra persona. Se dice entonces que el paciente ha recordado o ha desenterrado lo que había permanecido oculto durante mucho tiempo. El psicoterapeuta de la década de 1980, de 1990 y del nuevo milenio tiene en sus manos una forma de clasificación que no tenía el psicoterapeuta de 1920. ¿Podemos decir que el conocimiento de este último era inferior, inmaduro, involucionado, menos racional, erróneo? ¿O diremos que el psicoterapeuta de antaño y el actual habitan mundos distintos? Ahora veamos esta situación del lado del paciente. La persona que en una terapia actual llega a verse a sí misma como víctima de abuso en su infancia no se hubiera autopercibido como tal si tal terapia — que exige una clasificación — hubiera tenido lugar varias décadas atrás. En la biografía que logra reconstruir como rompecabezas a través de la psicoterapia el concepto de abuso no hubiera tenido existencia alguna, y en su lugar hubieran aparecido otras categorías que sí tenían peso explicativo en la época.

Arribamos entonces al siguiente planteamiento: ¡es nuestro autoconocimiento inmune a las transformaciones conceptuales? ¿Puedo tener un acceso epistémico puro a mi mundo interno? ¿ $\mathrm{O} \mathrm{mi}$ subjetividad y el sentido de lo que soy están condicionados por un marco conceptual que se va modelando históricamente? Si acepto que no hay un conocimiento incondicionado de mi mundo interno 
(como lo advirtió Kant), entonces cabe preguntarse si mi subjetividad y mi identidad personal son entidades dadas o si son, más bien, el producto de prácticas clasificatorias situadas de forma histórica y social que dan origen a esos moldes conceptuales a través de los cuales percibo el mundo externo e interno. Si denominamos artefacto a aquello cuya existencia depende de dichas prácticas es pertinente preguntarnos entonces si al final la subjetividad y el autoconocimiento no son también una clase de artefacto. Cuando, a través de una psicoterapia exitosa, una persona ha logrado recuperar una parte de su pasado que supuestamente había olvidado a causa del trauma, ¿qué es lo que tal recuerdo le ha devuelto? Pues, ese recuerdo supone una memorización, pero memorizar implica "describir acciones de una cierta forma” (Hacking, 1995). ¿Qué sucede entonces cuando la redescripción del pasado de una persona involucra ciertas categorías que no existían en el momento en que la acción ahora descrita tuvo lugar? Puesto de otra forma, el hábito del anacronismo historiográfico, que consiste en interpretar y explicar eventos del pasado a la luz de categorías actuales, ¿no se repite cuando lo que se reconstruye es una historia personal? Si las acciones humanas, tanto del presente como del pasado, pueden ser descritas en infinidad de formas de acuerdo a los marcos conceptuales dominantes en una época o cultura ¿hay una forma canónica de clasificarlas? ¿'Tenemos, cada uno de nosotros, un pasado que sea determinado, fijo, completo, invariable, no sujeto a reinterpretación y re-clasificación? ¿O lo que tenemos es una forma de recuerdo? Si sólo tenemos recuerdo, parecería entonces que lo que tenemos es una versión actual del pasado (porque recordar es ordenar valiéndose de conceptos de los que disponemos hoy).

Y si esto es así, entonces tendríamos que afirmar que el presente explica el pasado, porque lo reconstruimos con moldes conceptuales actuales. Pero a su vez la forma en la que armamos y rearmamos conceptualmente nuestro pasado condiciona el presente, porque nos 
da una imagen de nosotros mismos y de los demás; la imagen de nosotros que aparece a través del recuerdo — que implica clasificación- afecta e influye en nuestras creencias, en nuestras deliberaciones, en nuestras elecciones y en nuestras formas de actuar. Así, en pocas palabras, el presente nos abre la ventana a un pasado que reconstruimos y que, ya reconstruido, regresa a nuestro presente para transformarlo.

En este complejo proceso el discurso clasificador va ejerciendo todo su poder sobre el modo en que nos autopercibimos y nos comportamos. Ahora bien, hemos hablado del recuerdo que acontece en el transcurso de las psicoterapias, pero es necesario considerar que la narrativa acerca de nosotros mismos, de la clase de persona que somos y que son los demás, nuestro relato biográfico, no está en modo alguno constreñido a los ámbitos clínico-profesionales. El psiquiatra, el psicólogo, el psicopedagogo pueden clasificar nuestra conducta y dejar registro de ella en una historia clínica o en un archivo escolar, pero la dinámica clasificatoria que se desata a partir de esas formas de describirnos tiene una réplica en nuestra subjetividad, en la intimidad de una conciencia que comienza a pensarse a sí misma en los términos en los que ha sido descrita públicamente. Cada nueva taxonomía que emerge en los ámbitos teóricos ofrece una nueva forma de organizar un dominio de la experiencia y nos coloca en el nuevo mundo kuhniano, o, en los términos de Nelson Goodman, la nueva categoría deviene una clase relevante que trasciende esa instancia de investigación llegando a modelar nuestra percepción acerca de nuestro entorno y de nosotros mismos. De tal modo se puede enquistar una clasificación en nuestro discurso que, incluso en el caso en que se produzca un cambio científico importante que desplace a dicho concepto, la velocidad con la cual será también desplazado del lenguaje común será muchísimo más lenta. Por eso sucede lo que describíamos al comienzo de este ensayo: desapareció la histeria como una forma 
de diagnóstico, pero sobrevive aún en el imaginario popular. La realidad de la esquizofrenia es seriamente debatida y los antipsiquiatras pretendieron demolerla, pero esto no sacudió significativamente las ideas que se tenía de ella fuera de la esfera científica.

Hay, en mi opinión, un importante desfasaje entre la dinámica del cambio científico en las comunidades científicas y la dinámica del cambio científico al exterior de dichos ámbitos. Probablemente esto que estoy planteando no sea ninguna novedad, pero creo que este tipo de reflexión sobre cómo afectan las transformaciones conceptuales, taxonómicas y paradigmáticas a nuestra subjetividad, a nuestro autoconocimiento, a nuestra identidad, a nuestras elecciones y acciones puede ayudarnos a redimensionar un problema como el del cambio científico, que es, en definitiva, un cambio de vida.

Me parece, además, que es necesario tener en cuenta que, a la luz de los poderes causales que tienen las clasificaciones sobre los seres humanos, es importante comparar los efectos del cambio conceptual que se produce en las ciencias humanas con los efectos del cambio conceptual que tiene lugar en las ciencias naturales. Sobre este punto ya han advertido filósofos como Michel Foucault, Ian Hacking o Arnold Davidson: clasificar seres humanos es muy distinto que clasificar objetos naturales. Nombrar una conducta humana de determinada forma puede generar un efecto que no aparecería si dicha clasificación recayera sobre objetos que no tienen conciencia de ser nombrados y ordenados de una forma determinada. Las entidades del mundo natural pueden ser denominadas en infinidad de formas sin que este acto lingüístico afecte en lo más mínimo a su comportamiento (podríamos aplicarle el nombre de estrella a un planeta, o llamarlo satélite, o asteroide, o cometa, o cualquier otra denominación, por absurda o injuriosa que pudiera ser, pero esos cambios no podrían alterar el comportamiento natural de ese astro). En contraste con esa situación, cuando se clasifica a un ser humano de delirante 
(para poner un ejemplo) se puede generar un efecto en su comportamiento muy distinto al que se hubiera generado si hubiera sido clasificado como un genio. A diferencia de objetos no-concientes, el ser humano es sensible a las clasificaciones que se le aplican. Es muy distinto para una madre que le digan que su hijo es superdotado a que le digan que su hijo es retardado. Ambas conceptualizaciones tienen la capacidad de alterar el cristal a través del cual se percibe a nuestros semejantes y a nosotros mismos, con lo cual también modifican las relaciones familiares, los hábitos individuales y sociales, las expectativas, el trato, el modo de vida.

\section{Psicometría: un emblema clasificatorio de nuestra época}

Este último ejemplo es oportuno para reparar en una de las más dominantes tendencias científicas modeladoras en el siglo XX, ejemplo con el cual cerraré esta reflexión exploratoria. Hablar de un individuo con alta capacidad (como lo sugiere el término de superdotado) o de baja capacidad (retardado) responde a una forma de descripción y clasificación que desde hace poco más de 100 años ha adquirido un perfil epistémico sólido a la luz del desarrollo de una concepción de la inteligencia que se ha encarnado profundamente en nuestra cultura y que ha sostenido un conjunto de tecnologías sociales útil para la clasificación de los individuos y los grupos humanos (así como para decidir sobre sus destinos). Hay, en la actualidad, un modo riguroso de referirse a las capacidades humanas, valiéndose de un concepto que nos convierte en portadores de un número y un lugar en una escala de excelencia: el concepto de cociente intelectual, una idea que floreció desde el inicio del siglo con la evolución de lo que se conoce como tradición psicométrica.

El andamiaje conceptual-clasificatorio que trajo aparejada la visión cuantitativa de la inteligencia que subyace a tal noción ha reorganizado la forma de vida de quienes vivimos en nuestra cultura, 
ha introducido un nuevo modo de responder a un viejo enigma: ipor qué los seres humanos difieren tanto en sus formas de comportarse? Ya el hecho de responder esa pregunta enfatizando la capacidad intelectual es un rasgo distintivo del siglo XX (o, para ser más precisos, de las últimas dos décadas decimonónicas y de todo el siglo XX). Como intenté hacer ver en trabajos anteriores (Cfr. Christiansen, 2009) la pregunta por las diferencias individuales en el contexto previo al surgimiento de esta tradición psicométrica, que se inició con $\mathrm{F}$. Galton en la década de 1880, tenía una respuesta relacionada con el concepto de carácter y no con el de inteligencia. El carácter aludía a un conjunto de factores constitucionales y ambientales, fisiológicos y morales, innatos y adquiridos. Tener buen carácter no era equivalente a tener capacidad intelectual, sino a tener los valores que definían una conducta virtuosa (por ejemplo, ser perseverante, responsable, independiente, equilibrado, tener autocontrol, etc.) En ese contexto, la psicología era una ciencia del carácter ocupada de explicar cómo se formaban aquellas virtudes con valor individual y social.

Pero, décadas más tarde, la psicología tendió a seguir la dirección de aquellas investigaciones que le prometían un estatus experimental comparable al de otras ciencias, tal como lo ejemplificaba la psicofísica alemana. Poco a poco, la diversidad intelectual entre los individuos parecía explicar la diversidad en su éxito social, económico, escolar, laboral. El florecimiento de la idea de que la inteligencia puede medirse a través del uso de los tests mentales le proporcionó a la psicología una funcionalidad profesional de la cual había carecido. La psicometría parecía tener un vasto campo de aplicación para la administración de individuos y de grupos (la primera de ellas tuvo lugar en el ejército americano durante la primera guerra mundial, cuando se examinaron 1'700,000 soldados). ${ }^{1}$

${ }^{1}$ En lo que concierne al movimiento psicométrico, sigo el estudio realizado por López Cereso (1991). 
El paradigma psicométrico no sólo ha forjado la idea de que la inteligencia es una habilidad general que puede medirse mediante escalas mentales sino también la de que tiene una realidad fisiológica y hereditaria. La inteligencia es definida como una jerarquía de capacidades cognitivas determinadas de forma genética, que permite clasificar a los seres humanos a lo largo de un continuo de perfección intelectual; y que permite desarrollar políticas educacionales basadas en la aplicación de tests que miden el cociente intelectual, legitimando también la jerarquía socioeconómica que caracteriza a un orden social meritocrático.

Esta imagen de la naturaleza de la inteligencia y de su rol social ha sobrepasado completamente las fronteras académicas. Aun cuando varios pensadores han cuestionado con severidad esta concepción psicométrica, ello no ha detenido la filtración de su entramado conceptual en nuestras vidas cotidianas. Desde el punto de vista epistémico son muchas las preguntas que uno se puede hacer con respecto a la visión psicométrica de la inteligencia. A lo largo del siglo XX, algunos psicólogos, como los de la llamada Escuela de Chicago, liderados por L. Thurstone, criticaron la visión simplificadora de uno de los psicometristas más reconocidos, Ch. Spearman, quien defendía la estructura factorial de la inteligencia. En su lugar, proponían una imagen no jerárquica de la inteligencia entendida como compuesta por un número de factores independientes y primarios. Esta visión alternativa, que era igualmente compatible con datos estadísticos disponibles, dio impulso a la aparición de otros enfoques no jerárquicos de la inteligencia. Y la cuestión era que, si la inteligencia realmente consistía en un cierto número de habilidades mentales independientes - fuesen siete, como sostenía Thurstone, o 120, como sostenía J. P. Guilford-, entonces era imposible clasificar a los individuos a lo largo de una escala única de mérito social (Cfr. López Cereso, 1991). 
Sin embargo, a pesar de que no hay respuestas unánimes a preguntas como ¿̇se puede medir la inteligencia?, ¿qué es lo que miden los tests mentales? o ¿qué significa ser inteligente?, la tradición psicométrica sigue impactando con vehemencia en la sociedad y en nuestras vidas. Ante la gran diversidad humana y la necesidad de su ordenamiento, los psicometristas han hallado una forma de clasificar a los seres humanos de acuerdo con la posesión de un conjunto de habilidades deseables de forma social, y se han convertido en expertos "etiquetadores" de individuos que portan un número o puntaje que funciona como criterio para definir qué pueden hacer o de qué son capaces y de qué no.

No podemos dejar de recordar que la concepción psicométrica de la inteligencia ha sido inspiración para muchos de los movimientos eugenésicos del siglo $\mathrm{XX}$, al proporcionar parte de una arquitectura conceptual que permite pensar que la inteligencia está distribuida de forma desigual en la naturaleza y que la única forma de mejorar el nivel intelectual de un grupo, raza o nación es a través del control de las decisiones reproductivas de las parejas. El servicio prestado por el concepto de cociente intelectual para estos fines se puede ver, por ejemplo, en ciertas propuestas de promulgar de leyes de esterilización, como lo hizo en la década de 1970 William Shockley, premio Nobel de física, cuando sugirió la esterilización voluntaria $-\mathrm{y}$ compensada de forma económica - de aquellos que, luego de ser sometidos a tests mentales, demostraran tener un bajo cociente intelectual). Asimismo, la concepción psicométrica de la inteligencia le ha suministrado sustento científico a diversas iniciativas de leyes anti-inmigratorias, fundadas en la idea de que la mezcla de grupos humanos intelectualmente superiores con grupos humanos intelectualmente inferiores supondría, a largo plazo, una degeneración de la inteligencia nacional. Esta clase de argumentación alimentó, por ejemplo, las fuertes leyes anti-inmigrantes que se aprobaron en Esta- 
dos Unidos en 1924 para restringir, sobre todo, la entrada de migrantes procedentes de Europa oriental y del sur. Y podríamos, en verdad, hacer una larga lista de movimientos racistas y xenofóbicos para los cuales ha sido útil esta concepción hereditarista y biologicista de la inteligencia humana.

Ahora analicemos desde otra perspectiva el éxito conseguido por esta constelación de conceptos e ideas en la modelación de la vida de quienes habitamos en este universo psicometrista. Veamos más de cerca cómo ha funcionado en el área escolar. Uno de los más importantes antecedentes en tal sentido lo constituyó el hecho de que, entre 1944 y 1965, la aplicación de test mentales a niños de 11 ańos fungió como una ley nacional en Inglaterra, sancionada con la finalidad de regular el futuro acceso a la universidad. ¿Cuáles eran los supuestos implícitamente asumidos? Se puede decir que detrás de una medida semejante existía la convicción de que los tests mentales eran una herramienta útil para medir la clase de habilidades necesarias en una universidad; que tales habilidades tenían una base biológica que se mantenía constante a través de los años (desde la infancia hasta la adultez) y que el sistema educativo podía ser optimizado al darle las mejores oportunidades educativas a aquellos genéticamente aptos para satisfacer las expectativas del sistema. Aunque esta ley fue derogada luego de 21 años de vigencia, su espíritu sigue vivo en el sistema escolar actual, en especial en algunos reglamentos de admisión y de otorgamiento de becas, así como en ciertas posturas adoptadas con respecto a políticas educacionales específicas, como la de ofrecer educación compensatoria. En 1969 Arthur Jensen, adherido al determinismo mental galtoniano y a la tradición psicometrista, publicó un artículo donde sintetizaba, de alguna forma, el papel de esa visión de la inteligencia para tomar decisiones educacionales. Ese artículo se intitulaba “¿Cuánto podemos hacer para elevar el cociente intelectual y el éxito escolar?” (Jensen, cit. por López Cereso, 1991: 
423), a lo que Jensen respondía que, por vía educativa, no es mucho lo que se pude hacer. Su visión ha sido reforzada por aquellos que en la misma época, la década de 1970, intentaban mostrar la posibilidad de establecer mediciones neurológicas y fisiológicas de la inteligencia desde las primeras horas del nacimiento de una persona. Me refiero, por ejemplo, a Hans Eysenck y su equipo de investigación en el Instituto de Psiquiatría de la Universidad de Londres, quienes pretendían sostener que es posible predecir la inteligencia adulta en un bebé de 36 horas, al relacionar los niveles de cociente intelectual con el funcionamiento cerebral.

¿De qué otras maneras esta visión psicométrica de la inteligencia modela nuestras formas de vida? Veamos lo que ocurre en el ámbito laboral. El uso masivo de test mentales se ha convertido en una característica destacada en los procesos de selección del personal, y la idea que sustenta este hecho es la de que la medición del cociente intelectual de cada individuo genera una optimización de los recursos humanos. Ya en 1920, Henry Goddard, un psicometrista que popularizó el famoso test Binet-Simon en Estados Unidos, publicó un libro llamado Human Efficiency and Levels of Intelligence, donde afirmaba "La eficiencia social de un grupo de seres humanos depende de reconocer las limitaciones mentales de cada uno y de organizar la sociedad de modo que cada persona haga únicamente lo que su capacidad le permite" (Goddard, cit. por López Cereso, 1991: 421).

Pero esto no es todo lo que esa poderosísima sigla del cociente intelectual puede hacer, ya que incluso fenómenos como la delincuencia, la criminalidad y la enfermedad mental han comenzado a recibir una explicación en términos de inferioridad mental genéticamente basada. Nuestra subjetividad, nuestro autoconocimiento, nuestra identidad, parece atrapada entonces en esa lucha paradigmática, y sujeta, aunque con otra dinámica, a los vaivenes epistemológicos de la época y la cultura en la que nos ha tocado vivir. 


\section{Bibliografía}

Anscombe, E., 1959, Intention, Oxford, Blackwell.

Bazerman, C., 1987, "Codifying the social scientific style: The APA Publication Manual as a Behaviorist Rethoric", en Nelson, J., A. Megill y D. Closkey (eds.), The Rhetoric of the Human Sciences, Madison, University of Wisconsin Press.

Boyle, M., 1990, Schizophrenia: A Scientific Delusion?, Routledge, Londres.

Brozeck J., 1984, Explanations in the History of Psychology in the United States, Lewisburg, Bucknell University Press.

Christiansen, M. L., 2009, La arquitectura del destino. Reflexiones sobre la psicología del carácter desde la historiografía contextual, Guanajuato, Universidad de Guanajuato.

Danziger, K., 1984, "Towards a conceptual framework for a critical history of psychology", en Carpintero, H. y J. Peiro (eds.), Psychology in its Historical Context: Essays in Honour of Prof. Josef Brozek, Valencia, Monografías de la Revista de Historia de la Psicología.

Davidson, A., 1967, "Styles of Reasoning, Conceptual History and the Emergence of Psychiatry", en Mandelbaum, M., The Anatomy of Historical Knowledge, Baltimore, J. Hopkins University Press.

, 1984, "Radical Interpretation", en Inquiries into Truth and Interpretation, Oxford, Clarendon Press.

, 1987, "Sex and the Emergence of Sexuality", en Critical Inquiry, Chicago, The University of Chicago Press.

, 1990, "Closing up Corpses: Diseases of Sexuality and the Emergence of the Psychiatric Style of Reasoning", en Wallace, E. y J. Gach (eds.), Handbook for the History of Psychiatry.

Diagnostic and Statistical Manual of Mental Disorders, 1980, 1987 y 1994, $3^{a}$, ed., $3^{a}$ ed. revisada y $4^{a}$ ed., Washington D. C., American Psychiatric Association. 
Eysenck, H., 1973, The Inequality of Man, Londres, Temple Smith.

, 1979, The Structure and Measurement of Intelligence, Nueva York, Springer.

y L. J. Kamin, 1981, Intelligence, the Battle for the Mind, Amsterdam, Multimedia.

, 1982, A Model for Intelligence, Nueva York, Springer.

, 1988, "The Concept of Intelligence: Useful or Useless", en Intelligence, núm. 12.

Foucault, M., 1971, Madness and Civilization, Londres, Random House.

, 1973, The Birth of Clinic, Nueva York, Vintage Books.

, 1980, Power / Knowledge, Nueva York, Pantheon Books.

Goddard, H. H., 1920, Human Efficiency and Levels of Intelligence, Princeton, Princeton University Press.

Goldstein, J., 1987, Console and Classify: The French Psychiatric Profession in the Nineteenth Century, Nueva York, Cambridge University Press.

Hacking, I., 1982, "Biopower and the Avalanche of Numbers", en Humanities in Society, vol. 5, núms. 3 y 4.

, 1984, "Five Parables", en Rorty R., J. Scheneewind y K. Skinner (eds.), Philosophy in History: Essays on the Historiography of Philosophy, Cambridge, 1984.

, 1986, “The Invention of Split Personalities”, en Donogan A., A. Perovich y M. Wedin (eds.), Human Nature and Natural Knowledge, Dordrecht.

, 1986, “Making Up People”, en Heller, T. (ed.), 1986, Reconstructing Individualism: Autonomy, Individuality and the Self in Western Thought, California, Morton Sosna y Wellbery.

, 1988, "Double Consciousness in Britain, 1815-1875", en Dissociation, núm. 4. 
Press.

1990, The Taming of Chance, Cambridge, Cambridge University

, 1991, "Two Souls in One Body", en Critical Inquiry, núm. 17, Chicago, The University of Chicago Press.

, 1992a, "World-Making By Kind-Making: Child Abuse for Example”, en Douglas, M. y D. Hull (ed.), How Classification Works: N., Edinburgo, Goodman / The Social Sciences / Edinburgo University Press.

, 1992b, “'Style' for Historians and Philosophers", en Studies in History and Philosophy of Science, núm. 23.

, 1993, "Working in a New World: The Taxonomic Solution", en Horwich, P., World Changes. T. Kuhn and the Nature of Science, Cambridge, MIT Press.

, 1994, "The Looping Effect of the Human Kinds", en Sperber y Premack (eds.), Causal Cognition: A Multidisciplinary Approach.

, 1995, Rewriting the Soul, Princeton, Princeton University Press.

, 1998, Mad Travelers: Reflections on the Reality of Transient Mental Illness, Virginia, University Press of Virginia.

, 1999, Social Construction of What?, New Heaven, Harvard University Press.

Jensen, A. R., 1969, "How Much Can We Boost IQ and Scholastic Achievement?", en Genetics and education, Londres, Melthuen.

Kuhn, T., 1962, The Structure of Scientific Revolutions, Chicago, University of Chicago Press.

Laing, R., 1965, The Divided Self, Harmondsworth, Penguin. , 1967, The Politics of Experience, Harmondsworth, Penguin. , 1983, The Voice of Experience, Harmondsworth, Penguin.

Leary, D., 1987, “Telling likely stories: The rhetoric of the new psychology, 
1880-1920", Journal of the History of the Behavioral Sciences, núm. 23. López-Cereso, J., 1991, "Human Nature a Social Order: A Hundred Years of Psychometrics", en Journal of Social and Biological Structures, Oviedo, JAI Press.

Putnam, F., 1989, Diagnosis and Treatment of Multiple Personality Disorder, Nueva York, The Guilford Press.

Reznek, L., 1987, The Nature of Disease, Nueva York, Routledge \& Kegan Paul. , 1991, The Philosophical defense of Psychiatry, Nueva York, Routledge. Scheff, T., 1975, Labeling Madness, Nueva York, Prentice Hall.

Spearman, C., 1923, "Recent Contributions to the theory of Two Factors", The British Journal of Psychology, vol. XIII, núms. 26-30.

Szasz, T., 1972, The Myth of Mental Disease, Londres, Paladin. , 1979, Schizophrenia, Oxford, Oxford University Press. , 1987, Insanity, Nueva York, John Wiley \& Sons.

Thurstone, L., 1924, The Nature of Intelligence, Nueva York, Harcourt, Brace and Co.

Tyrer y Steinberg, D., 1987, Models for Mental Disorder, Chichester, John Wiley \& Sons.

Wilbur, C., 1986, "Psychoanalysis and Multiple Personality Disorder MPD”, en Braun, B. (ed.), Treatment of MPD, Washington, American Psychiatric Press.

y R. Kluft, 1989, MPD, en Treatment of Psychiatric Disorders, núm. 3, Washington D. C., APA. 